\section{TATRA \\ MOUNTaiNS \\ Mathematical Publications}

DOI: $10.2478 /$ tmmp-2019-0017

Tatra Mt. Math. Publ. 74 (2019), 35-46

\title{
I-COMPLETENESS IN FUNCTION SPACES
}

\author{
Amar Kumar BanerJee — Apurba BanerJee \\ Department of Mathematics, The University of Burdwan, Burdwan, West Bengal, INDIA
}

\begin{abstract}
In this paper, we have studied the idea of ideal completeness of function spaces $Y^{X}$ with respect to pointwise uniformity and uniformity of uniform convergence. Further, involving topological structure on $X$, we have obtained relationships between the uniformity of uniform convergence on compacta on $Y^{X}$ and uniformity of uniform convergence on $Y^{X}$ in terms of $I$-Cauchy condition and $I$-convergence of a net. Also, using the notion of a $k$-space, we have given a sufficient condition for $C(X, Y)$ to be ideal complete with respect to the uniformity of uniform convergence on compacta.
\end{abstract}

\section{Introduction}

The idea of convergence of a real sequence was extended to statistical convergence by H. Fast [5] (see also I. J. S choen berg [17]) as follows:

If $\mathbb{N}$ denotes the set of all natural numbers and $K \subset \mathbb{N}$, then $K_{n}$ denotes the set $\{k \in K: k \leq n\}$ and $\left|K_{n}\right|$ stands for the cardinality of the set $K_{n}$. The natural density of the set $K$ is defined by $d(K)=\lim _{n \rightarrow \infty} \frac{\left|K_{n}\right|}{n}$, provided the limit exists.

A sequence $\left\{x_{n}\right\}$ of points in the real number space is said to be statistically convergent to $x_{0}$ if for arbitrary $\epsilon>0$ the set $K(\epsilon)=\left\{k \in \mathbb{N}:\left|x_{k}-x_{0}\right| \geq \epsilon\right\}$ has natural density zero. A lot of works have been done so far on such convergence and its topological consequences after the initial works by T. Š a lát [16]. However, if one considers the concept of nets instead of sequences, the above approach does not seem to be appropriate because of the absence of any idea of density in an arbitrary directed set. Instead, it seems more appropriate to follow the more general approach of ideal convergence [8]. In [8] (see also [9]), a generalization of the notion of statistical convergence was proposed as follows:

(C) 2019 Mathematical Institute, Slovak Academy of Sciences.

2010 Mathematics Subject Classification: Primary 54A20; Secondary 40A35, 54E15. Keywords: ideal, filter, uniform space, $I$-Cauchy condition, $I$-convergence, ideal completeness.

Licensed under the Creative Commons Attribution-NC-ND4.0 International Public License. 
A subcollection $I \subset 2^{\mathbb{N}}$ is called an ideal if

(i) $A, B \in I$ implies $A \cup B \in I$ and

(ii) $A \in I, B \subset A$ imply $B \in I$.

$I$ is called non-trivial ideal if $I \neq\{\Phi\}$ and $\mathbb{N} \notin I$. I is called admissible if it contains all singletons. If $I$ is a proper non-trivial ideal, then the family of sets $F(I)=\{M \subset \mathbb{N}: \mathbb{N} \backslash M \in I\}$ is a filter on $\mathbb{N}$ and it is called the filter associated with the ideal $I$ of $\mathbb{N}$. It is easy to check that the family $I_{d}=\{A \subset \mathbb{N}: d(A)=0\}$ forms a non-trivial admissible ideal of $\mathbb{N}$.

A sequence $\left\{x_{n}\right\}$ of real numbers is said to be $I$-convergent to $x_{0} \in X$ (in short, $x_{0}=I-\lim _{n \rightarrow \infty} x_{n}$ ) if $K(\epsilon) \in I$ for each $\epsilon>0$, where $K(\epsilon)=\{k \in \mathbb{N}$ : $\left.\left|x_{k}-x_{0}\right| \geq \epsilon\right\}$.

B. K. Lahiri and P. D as [12] extended the idea of $I$-convergence to an arbitrary topological space and observed that few basic properties related to ideal convergence are also preserved like ordinary convergence in a topological space. They also introduced [13] the idea of $I$-convergence of nets in a topological space and examined how far it affects the basic properties. Later, P. D a s and S. K. Gh o s a 1 [4 introduced the idea of $I$-Cauchy nets in a uniform space and formulated two equivalent forms of $I$-Cauchy condition of a net in a uniform space. Also, they proved that every $I$-convergent net in a uniform space with respect to the uniform topology satisfies $I$-Cauchy condition. Further, they have given a sufficient condition for uniform spaces to be complete in terms of $I$-convergence of $I$-Cauchy nets.

In this paper, we have studied the idea of ideal completeness of a uniform space and have shown a sufficient condition for a subspace of product uniform space with respect to the pointwise uniformity to be ideal complete. Again, we have given a necessary and sufficient condition for a product uniform space with respect to the uniformity of uniform convergence to be ideal complete. Also, we have obtained that if a uniform space $(Y, \mathcal{U})$ is ideal complete, then so are $\left(Y^{X}, \mathcal{U}_{u}\right)$ and $\left(C(X, Y), \mathcal{U}_{u}\right)$, where $X$ is a non-empty set and $\left(Y^{X}, \mathcal{U}_{u}\right)$ is the product uniform space with respect to the uniformity of uniform convergence $\mathcal{U}_{u}$, and $C(X, Y)$ is the space of all continuous functions from $X$ to $Y$.

Further, involving topology on $X$ in the function space, we have shown separately that a net $\left\{f_{\lambda}: \lambda \in \Lambda\right\}$ in $\left(Y^{X}, \mathcal{U}_{k}\right)$, where $\mathcal{U}_{k}$ is the uniformity of uniform convergence on compacta, is an $I$-Cauchy net if and only if for each compact subset $K$ of $X,\left\{\left.f_{\lambda}\right|_{K}: \lambda \in \Lambda\right\}$ is $I$-Cauchy in the uniformity of uniform convergence on $K$ and the same result has been given in case of $I$-convergence of a net in $\left(Y^{X}, \tau_{\mathcal{U}_{k}}\right)$ where $\tau_{\mathcal{U}_{k}}$ is the topology of uniform convergence on compacta. Finally, applying the idea of a $k$-space and using the preceding results, we have shown that if $X$ is a $k$-space and $(Y, \mathcal{U})$ is ideal complete, then $C(X, Y)$ is ideal complete in the uniformity of uniform convergence on compacta. 


\section{I-COMPLETENESS IN FUNCTION SPACES}

\section{Ideal completeness in function spaces}

Let $(Y, \tau)$ be a topological space and $X$ be a non-empty set. Let $Y^{X}$ be endowed with the Tychonoff product topology. We say a subcollection $\mathcal{F} \subset Y^{X}$ has the topology of pointwise convergence (or, the pointwise topology) if and only if it is provided with the subspace topology induced by the Tychonoff product topology on $Y^{X}$. Let $(Y, \mathcal{U})$ be a uniform space which we will write sometimes simply as $Y$. It may be recalled that for any point $x$ in a uniform space $(Y, \mathcal{U})$, the collection $\{U[x]: x \in Y\}$ [where $U[x]=\{y \in Y:(x, y) \in U\}]$ forms a local neighbourhood base at $x$. The corresponding topology $\tau_{\mathcal{U}}$ is called the uniform topology on $Y$. By an open set in $Y$ we shall always mean an open set in the uniform topology in $Y$.

Let $(D, \geq)$ be a directed set and $I$ be a non-trivial ideal of $D$. A net in $Y$ will be denoted by $\left\{s_{\alpha}: \alpha \in D\right\}$ or simply by $\left\{s_{\alpha}\right\}$, when there is no confusion about $D$. For $\alpha \in D$, let

$$
M_{\alpha}=\{\beta \in D: \beta \geq \alpha\} .
$$

Then, the collection $F_{0}=\left\{A \subset D: A \supset M_{\alpha}\right.$, for some $\left.\alpha \in D\right\}$ forms a filter in $D$. Let $I_{0}=\left\{B \subset D: D \backslash B \in F_{0}\right\}$. Then, $I_{0}$ is a non-trivial ideal of $D$.

Definition 2.1 ([13]). A non-trivial ideal $I$ of $D$ will be called $D$-admissible if $M_{\alpha} \in F(I)$ for all $\alpha \in D$.

Definition 2.2 (13]). A net $\left\{s_{\alpha}: \alpha \in D\right\}$ in $(Y, \mathcal{U})$ is said to be $I$-convergent to $x_{0} \in Y$ if for any open set $U$ in $\left(Y, \tau_{\mathcal{U}}\right)$ containing $x_{0},\left\{\alpha \in D: s_{\alpha} \notin U\right\} \in I$.

Definition $2.3(4])$. A net $\left\{s_{\alpha}: \alpha \in D\right\}$ in a uniform space $(Y, \mathcal{U})$ is said to be $I$-Cauchy if for any $U \in \mathcal{U}$, there exists a $\beta \in D$ such that $\left\{\alpha \in D:\left(s_{\alpha}, s_{\beta}\right) \notin U\right\} \in I$.

It is easy to check that when $I=I_{0}$, the definition of $I$-Cauchy condition of a net coincides with the usual Cauchy condition.

We know two equivalent forms of $I$-Cauchy condition of a net in a uniform space which are stated below.

Theorem 2.1 ([4]). For a net $\left\{s_{\alpha}: \alpha \in D\right\}$ in a uniform space $(Y, \mathcal{U})$, the following conditions are equivqlent:

(1) $\left\{s_{\alpha}: \alpha \in D\right\}$ is an I-Cauchy net.

(2) For every $U \in \mathcal{U}$ there exists $A \in I$ such that $\alpha, \beta \notin A$ implies $\left(s_{\alpha}, s_{\beta}\right) \in U$.

(3) For every $U \in \mathcal{U},\left\{\beta \in D: E_{\beta}(U) \notin I\right\} \in I$, where

$$
E_{\beta}(U)=\left\{\alpha \in D:\left(s_{\alpha}, s_{\beta}\right) \notin U\right\} .
$$

Throughout the paper, we assume that $\Lambda$ is a directed set, $(Y, \tau)$ is a topological space and $X$ is a non-empty set, $Y^{X}$ is endowed with the Tychonoff product topology and $\mathcal{F} \subset Y^{X}$ has the pointwise topology (i.e., subspace topology induced by the Tychonoff product topology on $Y^{X}$ ) unless otherwise stated. 
Theorem 2.2 ([2]). If $\mathcal{F}$ has the pointwise topology, then a net $\left\{f_{\lambda}: \lambda \in \Lambda\right\}$ is I-convergent to $f$ in $\mathcal{F}$ if and only if the net $\left\{f_{\lambda}(x): \lambda \in \Lambda\right\}$ is I-convergent to $f(x)$ in $\pi_{x}(\mathcal{F})$ for each $x \in X$, where $I$ is a non-trivial ideal of the directed set $\Lambda$ and $\pi_{x}$ is the $x$ th projection map from $Y^{X}$ onto $Y$.

Pro of. Since $\pi_{x}\left(f_{\lambda}\right)=f_{\lambda}(x)$ for $x \in X$, the proof follows from [2, Theorem 3.4].

We turn now to the discussion of defining a uniformity on the product of uniform spaces, subject to the obvious restriction that the topology of such a uniformity should be the product topology.

First, we recall the following definition.

Definition 2.4 ([18]). If $X_{\alpha}$ is a set for each $\alpha \in \mathcal{A}$ and $X=\prod X_{\alpha}$, the $\alpha$ th biprojection is the map

$$
P_{\alpha}: X \times X \rightarrow X_{\alpha} \times X_{\alpha}
$$

defined by $P_{\alpha}(x, y)=\left(\pi_{\alpha}(x), \pi_{\alpha}(y)\right)$, where $\pi_{\alpha}$ is the $\alpha$ th projection mapping from $X$ onto $X_{\alpha}$.

Theorem 2.3 ([18]). If $\mathcal{U}_{\alpha}$ is a diagonal uniformity on $X_{\alpha}$, for each $\alpha \in \mathcal{A}$, then the sets

$$
P_{\alpha_{1}}^{-1}\left(U_{\alpha_{1}}\right) \cap P_{\alpha_{2}}^{-1}\left(U_{\alpha_{2}}\right) \cap \cdots \cap P_{\alpha_{n}}^{-1}\left(U_{\alpha_{n}}\right),
$$

where $U_{\alpha_{i}} \in \mathcal{U}_{\alpha_{i}}$, for $i=1,2, \ldots, n$, form a base for a uniformity $\mathcal{U}_{p}$ on $\prod X_{\alpha}$ which is called the product uniformity on $\prod X_{\alpha}$ and whose associated topology is the product topology on $\prod X_{\alpha}$.

Now, let us assume that $(Y, \mathcal{U})$ is a uniform space.

Definition 2.5 ([18]). The product uniformity $\mathcal{U}_{p}$ on $Y^{X}$ is called the uniformity of pointwise convergence or the pointwise uniformity.

Note that the topology associated with the pointwise uniformity on $Y^{X}$ is, of course, the pointwise topology.

Theorem 2.4. $\left\{f_{\lambda}: \lambda \in \Lambda\right\}$ is an I-Cauchy net in $Y^{X}$ with the pointwise uniformity if and only if $\left\{f_{\lambda}(x): \lambda \in \Lambda\right\}$ is an I-Cauchy net in $Y$ for each $x \in X$, where $I$ is a non-trivial ideal of $\Lambda$.

Proof. Let $\left\{f_{\lambda}: \lambda \in \Lambda\right\}$ be an $I$-Cauchy net in $Y^{X}$ with the pointwise uniformity. Then, for every member of $\mathcal{U}_{p}$ of the form $P_{x}^{-1}(U)$, where $U \in \mathcal{U}$, there exists $B \in I$ such that $\alpha, \beta \notin B$ implies $\left(f_{\alpha}, f_{\beta}\right) \in P_{x}^{-1}(U)$, i.e., $P_{x}\left(f_{\alpha}, f_{\beta}\right) \in U$, i.e., $\left(f_{\alpha}(x), f_{\beta}(x)\right) \in U$ and hence by Theorem 2.1, it follows that $\left\{f_{\alpha}(x): \alpha \in \Lambda\right\}$ is an $I$-Cauchy net in $Y$ for each $x \in X$. 


\section{I-COMPLETENESS IN FUNCTION SPACES}

Conversely, suppose $\left\{f_{\lambda}(x): \lambda \in \Lambda\right\}$ is an $I$-Cauchy net in $Y$ for each $x \in X$. Hence by Theorem 2.1, for each $U \in \mathcal{U}$ and for each $x \in X$, there exists $A_{0} \in I$ such that $\alpha, \beta \notin A_{0}$ implies $\left(f_{\alpha}(x), f_{\beta}(x)\right) \in U$. Let us choose a member $U \in$ $\mathcal{U}_{p}$. Then, $U$ has the form $P_{x_{1}}^{-1}\left(U_{1}\right) \cap P_{x_{2}}^{-1}\left(U_{2}\right) \cap \cdots \cap P_{x_{n}}^{-1}\left(U_{n}\right)$, where $U_{i} \in \mathcal{U}$ for all $i=1,2, \ldots, n$ and $x_{1}, x_{2}, \ldots, x_{n} \in X$. Now, for $U_{1}, U_{2}, \ldots, U_{n} \in \mathcal{U}$ and $x_{1}, x_{2}, \ldots, x_{n} \in X$ there exist $A_{1}, A_{2}, \ldots, A_{n} \in I$ such that $\lambda_{1}, \lambda_{2} \notin A_{i}$ implies $\left(f_{\lambda_{1}}\left(x_{i}\right), f_{\lambda_{2}}\left(x_{i}\right)\right) \in U_{i}$ for $i=1,2, \ldots, n$. Let $A=\bigcup_{i=1}^{n} A_{i}$. Then, $A \in I$. Now, $\alpha, \beta \notin A$ implies $\left(f_{\alpha}\left(x_{i}\right), f_{\beta}\left(x_{i}\right)\right) \in U_{i}$ for each $i=1,2, \ldots, n$, which in turn implies that $P_{x_{i}}\left(f_{\alpha}, f_{\beta}\right) \in U_{i}$ for each $i=1,2, \ldots, n$, i.e., $\left(f_{\alpha}, f_{\beta}\right) \in P_{x_{i}}^{-1}\left(U_{i}\right)$ for each $i=1,2, \ldots, n$. Thus, $\alpha, \beta \notin A$ implies $\left(f_{\alpha}, f_{\beta}\right) \in \bigcap_{i=1}^{n} P_{x_{i}}^{-1}\left(U_{i}\right)=U \in \mathcal{U}_{p}$. Hence, again applying Theorem 2.1, we get that $\left\{f_{\lambda}: \lambda \in \Lambda\right\}$ is an $I$-Cauchy net in $Y^{X}$.

We define below the notion of ideal completeness of a uniform space in the same manner as that of a uniform space to be complete.

Definition 2.6. A uniform space $(Y, \mathcal{U})$ is said to be ideal complete if every net $\left\{s_{\alpha}: \alpha \in \Lambda\right\}$ in $(Y, \mathcal{U})$, which is $I$-Cauchy in $(Y, \mathcal{U})$, is $I$-convergent in $\left(Y, \tau_{\mathcal{U}}\right)$, where $I$ is a non-trivial ideal of $\Lambda$ and $\tau_{\mathcal{U}}$ is the uniform topology on $Y$ corresponding to the uniformity $\mathcal{U}$ on $Y$.

Theorem 2.5. Let $\mathcal{F} \subset Y^{X}$ be a function space with the pointwise uniformity. Let $\Lambda$ be a directed set and $I$ be a non-trivial ideal of $\Lambda$. Then, $\mathcal{F}$ is ideal complete if

(1) $\mathcal{F}$ is pointwise closed (i.e., $\mathcal{F}$ is closed in the pointwise topology on $Y^{X}$ ),

(2) $\pi_{x}(\mathcal{F})=\{f(x): f \in \mathcal{F}\}$ is ideal complete in $Y$ for each $x \in X$.

Proof. Let $\left\{f_{\lambda}: \lambda \in \Lambda\right\}$ be an $I$-Cauchy net in $\mathcal{F}$. Then for each $x \in X$, $\left\{\pi_{x}\left(f_{\lambda}\right): \lambda \in \Lambda\right\}$ is an $I$-Cauchy net in $\pi_{x}(\mathcal{F})$. Since $\pi_{x}(\mathcal{F})$ is ideal complete, so by definition, $\left\{\pi_{x}\left(f_{\lambda}\right): \lambda \in \Lambda\right\}$ is $I$-convergent to some $f(x) \in \pi_{x}(\mathcal{F})$ and this holds for each $x \in X$. Thus, we see that $\left\{\pi_{x}\left(f_{\lambda}\right): \lambda \in \Lambda\right\}$ is $I$-convergent to $f(x)$ in $\pi_{x}(\mathcal{F})$ for each $x \in X$. Now, applying Theorem 2.2 , we get that $\left\{f_{\lambda}: \lambda \in \Lambda\right\}$ is $I$-convergent to $f$ in $Y^{X}$. Since $\mathcal{F}$ is pointwise closed, so we have $f \in \mathcal{F}$. Hence the result follows.

We know that pointwise $I$-limit of continuous functions (on the real line, say) need not be continuous, so that $C(X, Y)$, the space of all continuous functions from $X$ to $Y$, is not always ideal complete in the uniformity of pointwise convergence.

The uniformity of pointwise convergence and its topology occupy one end of the spectrum of structures used to make function spaces out of collections of functions. At the other end, sit the uniformity of uniform convergence and its topology which we recapitulate below. 
Definition 2.7 ([18]). If $Y$ has a uniformity $\mathcal{U}$, the family of sets of the form

$$
E_{U}=\left\{(f, g) \in Y^{X} \times Y^{X}:(f(x), g(x)) \in U \text { for each } x \in X\right\}
$$

for $U \in \mathcal{U}$, form a base for a uniformity $\mathcal{U}_{u}$ on $Y^{X}$ called the uniformity of uniform convergence. Its associated topology, $\tau_{\mathcal{U}_{u}}$, is the topology of uniform convergence.

Definition 2.8. If a net $\left\{f_{\lambda}: \lambda \in \Lambda\right\}$ in $Y^{X}$ is $I$-convergent to $f \in Y^{X}$ in the topology of uniform convergence, we say $\left\{f_{\lambda}: \lambda \in \Lambda\right\}$ is uniformly $I$-convergent to $f$, where $I$ is a non-trivial ideal of $\Lambda$.

Definition 2.9. If a net $\left\{f_{\lambda}: \lambda \in \Lambda\right\}$ in $Y^{X}$ is $I$-Cauchy in the uniformity of uniform convergence, then we call $\left\{f_{\lambda}: \lambda \in \Lambda\right\}$ uniformly $I$-Cauchy, where $I$ is a non-trivial ideal of $\Lambda$.

The next theorem provides a relationship between pointwise $I$-convergence and uniform $I$-convergence of a net and subsequently gives a necessary and sufficient condition for a product uniform space with respect to the uniformity of uniform convergence to be ideal complete.

Theorem 2.6. A net $\left\{f_{\lambda}: \lambda \in \Lambda\right\}$ in $\left(Y^{X}, \tau_{\mathcal{H}_{u}}\right)$ is uniformly I-convergent to $f$ if and only if

(1) the net $\left\{f_{\lambda}: \lambda \in \Lambda\right\}$ is uniformly I-Cauchy in $\left(Y^{X}, \mathcal{U}_{u}\right)$ and

(2) the net $\left\{f_{\lambda}(x): \lambda \in \Lambda\right\}$ is I-convergent to $f(x)$ in $\left(Y, \tau_{\mathcal{U}}\right)$ for each $x \in X$ [i.e., the net $\left\{f_{\lambda}: \lambda \in \Lambda\right\}$ is pointwise I-convergent to $f$ in $\left.\left(Y^{X}, \tau_{\mathcal{U}_{u}}\right)\right]$, where $I$ is a non-trivial ideal of $\Lambda$.

Proof. Let $\left\{f_{\lambda}: \lambda \in \Lambda\right\}$ be a net in $\left(Y^{X}, \mathcal{U}_{u}\right)$ which is uniformly $I$-convergent to $f \in Y^{X}$. So, by [4. Theorem 2], it follows that $\left\{f_{\lambda}: \lambda \in \Lambda\right\}$ is uniformly $I$-Cauchy. Now, by definition of $I$-convergence of a net, for any $U \in \mathcal{U}$ we have the set

$$
\left\{\lambda \in \Lambda: f_{\lambda} \notin E_{U}[f]\right\} \in I
$$

where $E_{U}[f]=\left\{g \in Y^{X}:(f, g) \in E_{U}\right\}=\left\{g \in Y^{X}:(f(x), g(x)) \in U\right.$ for each $x \in X\}$. This implies the set $\left\{\lambda \in \Lambda: f_{\lambda} \in E_{U}[f]\right\} \in F(I)$, where $F(I)$ is the filter associated with the ideal $I$. Let $A=\left\{\lambda \in \Lambda: f_{\lambda} \in E_{U}[f]\right\}$. Then, $\lambda_{0} \in A$ implies $f_{\lambda_{0}} \in E_{U}[f]$, i.e., $\left(f, f_{\lambda_{0}}\right) \in E_{U}$ and so, $\left(f(x), f_{\lambda_{0}}(x)\right) \in U$ for each $x \in X$, i.e., $f_{\lambda_{0}}(x) \in U[f(x)]$ for each $x \in X$. Now, let $x_{0} \in X$ be an arbitrary element. Then, if $A_{0}=\left\{\alpha \in \Lambda: f_{\alpha}\left(x_{0}\right) \in U\left[f\left(x_{0}\right)\right]\right\}$, we see that $A \subset A_{0}$, and since $A \in F(I)$, so we have by definition of a filter $A_{0} \in F(I)$ as well. So, the set $\left\{\alpha \in \Lambda: f_{\alpha}\left(x_{0}\right) \notin U\left[f\left(x_{0}\right)\right]\right\} \in I$, i.e., the net $\left\{f_{\lambda}\left(x_{0}\right): \lambda \in \Lambda\right\}$ is $I$ -convergent to $f\left(x_{0}\right)$ in $\left(Y, \tau_{\mathcal{U}}\right)$. Hence, we conclude that the net $\left\{f_{\lambda}(x): \lambda \in \Lambda\right\}$ is $I$-convergent to $f(x)$ in $\left(Y, \tau_{\mathcal{U}}\right)$ for each $x \in X$. 


\section{I-COMPLETENESS IN FUNCTION SPACES}

Conversely, let the net $\left\{f_{\lambda}: \lambda \in \Lambda\right\}$ in $Y^{X}$ be pointwise $I$-convergent to $f$, i.e., the net $\left\{f_{\lambda}(x): \lambda \in \Lambda\right\}$ is $I$-convergent to $f(x)$ in $\left(Y, \tau_{\mathcal{U}}\right)$ for each $x \in X$ and $\left\{f_{\lambda}: \lambda \in \Lambda\right\}$ is uniformly $I$-Cauchy in $\left(Y^{X}, \mathcal{U}_{u}\right)$. To show that $\left\{f_{\lambda}: \lambda \in \Lambda\right\}$ is uniformly $I$-convergent to $f$ in $\left(Y^{X}, \tau_{\mathcal{U}_{u}}\right)$, we are to show that for any $U \in \mathcal{U}$ the set $\left\{\lambda \in \Lambda: f_{\lambda} \notin E_{U}[f]\right\} \in I$. Now, for each $x \in X$ we have the set $\left\{\lambda \in \Lambda: f_{\lambda}(x) \notin U[f(x)]\right\} \in I$, i.e., $\left\{\lambda \in \Lambda: f_{\lambda}(x) \in U[f(x)]\right\} \in F(I)$, where $F(I)$ is the filter associated with the ideal $I$. For each $x \in X$ let us call the set $B_{x}=\left\{\lambda \in \Lambda: f_{\lambda}(x) \in U[f(x)]\right\}$. Then, $B_{x} \in F(I)$ for each $x \in X$. Now, choose a symmetric $V \in \mathcal{U}$ such that $V \circ V \subset U$. For each $x \in X$ let us call $C_{x}=\left\{\lambda \in \Lambda: f_{\lambda}(x) \in V[f(x)]\right\}$. Then, again on the basis of the condition (2), we have $C_{x} \in F(I)$ for each $x \in X$. Since $\left\{f_{\lambda}: \lambda \in \Lambda\right\}$ is uniformly $I$-Cauchy in $\left(Y^{X}, \mathcal{U}_{u}\right)$, so by Theorem 2.1 we have that for $V \in \mathcal{U}$ there exists $A \in I$ such that $\alpha, \beta \notin A$ implies $\left(f_{\alpha}, f_{\beta}\right) \in E_{V}$. We will prove that for any arbitrary $x_{0} \in X$, $A^{c} \subset B_{x_{0}}$. Now, since $A^{c}, C_{x_{0}} \in F(I)$, so $A^{c} \cap C_{x_{0}} \neq \phi$. Let us take $\alpha_{0} \in A^{c} \cap C_{x_{0}}$. Then, for any $\alpha \in A^{c}$ we have $\left(f_{\alpha_{0}}, f_{\alpha}\right) \in E_{V}$, i.e., $\left(f_{\alpha_{0}}(x), f_{\alpha}(x)\right) \in V$ for each $x \in X$. Hence, in particular, $\left(f_{\alpha_{0}}\left(x_{0}\right), f_{\alpha}\left(x_{0}\right)\right) \in V$. Again, since $\alpha_{0} \in C_{x_{0}}$, so, $f_{\alpha_{0}}\left(x_{0}\right) \in V\left[f\left(x_{0}\right)\right]$, i.e., $\left(f\left(x_{0}\right), f_{\alpha_{0}}\left(x_{0}\right)\right) \in V$. Thus, we get $\left(f\left(x_{0}\right), f_{\alpha}\left(x_{0}\right)\right) \in$ $V \circ V \subset U$, i.e., $f_{\alpha}\left(x_{0}\right) \in U\left[f\left(x_{0}\right)\right]$, i.e., $\alpha \in B_{x_{0}}$. Hence, $A^{c} \subset B_{x_{0}}$. Since $x_{0} \in X$ has been chosen arbitrarily, so we conclude that $A^{c} \subset \bigcap_{x \in X} B_{x}$. This in turn implies that $\bigcap_{x \in X} B_{x} \in F(I)$. Now, we see that $\bigcap_{x \in X} B_{x}=\left\{\lambda \in \Lambda: f_{\lambda}(x) \in\right.$ $U[f(x)]$ for each $x \in X\}=\left\{\lambda \in \Lambda: f_{\lambda} \in E_{U}[f]\right\}$. Thus in turn, we have proved that $\left\{\lambda \in \Lambda: f_{\lambda} \notin E_{U}[f]\right\} \in I$. Hence the result follows.

TheOREM 2.7. If a uniform space $(Y, \mathcal{U})$ is ideal complete, then so are

(1) $\left(Y^{X}, \mathcal{U}_{u}\right)$,

(2) $\left(C(X, Y), \mathcal{U}_{u}\right)$, where $C(X, Y)$ is the space of all continuous functions from $X$ to $Y$ with the uniformity of uniform convergence $\mathcal{U}_{u}$.

P r o of. (1): Let a net $\left\{f_{\lambda}: \lambda \in \Lambda\right\}$ be uniformly $I$-Cauchy in $\left(Y^{X}, \mathcal{U}_{u}\right)$. Then, the net $\left\{f_{\lambda}(x): \lambda \in \Lambda\right\}$ is $I$-Cauchy in $(Y, \mathcal{U})$ for each $x \in X$. Hence, $\left\{f_{\lambda}(x): \lambda \in \Lambda\right\}$ is $I$-convergent to some $f(x) \in Y$, since $(Y, \mathcal{U})$ is ideal complete space. By previous result, the function $f \in Y^{X}$ defined by $f(x)=I$ - $\lim f_{\lambda}(x)$ for each $x \in X$ is uniform $I$-limit of the net $\left\{f_{\lambda}: \lambda \in \Lambda\right\}$. Thus, $\left(Y^{X}, \mathcal{U}_{u}\right)$ is ideal complete.

(2): It has been proved in [18, Theorem 42.10] that $\left(C(X, Y), \mathcal{U}_{u}\right)$ is a closed subspace of $\left(Y^{X}, \mathcal{U}_{u}\right)$.

First, we prove that if $(M, \mathcal{D})$ is a uniform space and $N \subset M$, then an $I$-Cauchy net $\left\{x_{\lambda}: \lambda \in \Lambda\right\}$ in $\left(N, \mathcal{D}_{N}\right)$ is also $I$-Cauchy in $(M, \mathcal{D})$, where $I$ is a non-trivial ideal of $\Lambda$ and $\mathcal{D}_{N}$ is the relative uniformity induced on $N$ by $\mathcal{D}$. Now, since $\left\{x_{\lambda}: \lambda \in \Lambda\right\}$ is an $I$-Cauchy net in $\left(N, \mathcal{D}_{N}\right)$, so for each $D_{N} \in \mathcal{D}_{N}$ there exists some $\lambda_{0} \in \Lambda$ such that the set $\left\{\lambda \in \Lambda:\left(x_{\lambda}, x_{\lambda_{0}}\right) \notin D_{N}\right\} \in I$. Now, $D_{N}=$ $D \cap(N \times N)$ where $D \in \mathcal{D}$ and for each $D \in \mathcal{D}$ there corresponds a $D_{N} \in \mathcal{D}_{N}$. 
We also note that $\left\{\lambda \in \Lambda:\left(x_{\lambda}, x_{\lambda_{0}}\right) \notin D\right\} \subset\left\{\lambda \in \Lambda:\left(x_{\lambda}, x_{\lambda_{0}}\right) \notin D_{N}\right\}$. Hence, $\left\{\lambda \in \Lambda:\left(x_{\lambda}, x_{\lambda_{0}}\right) \notin D\right\} \in I$. So, for each $D \in \mathcal{D}$ there is some $\lambda_{0} \in \Lambda$ such that the set $\left\{\lambda \in \Lambda:\left(x_{\lambda}, x_{\lambda_{0}}\right) \notin D\right\} \in I$. Hence, $\left\{x_{\lambda}: \lambda \in \Lambda\right\}$ becomes an I-Cauchy net in $(M, \mathcal{D})$.

Secondly, we show that if $(M, \mathcal{D})$ is an ideal complete uniform space and $N$ is a closed subset of $\left(M, \tau_{\mathcal{D}}\right)$, then $\left(N, \mathcal{D}_{N}\right)$ becomes an ideal complete space.

Since an $I$-Cauchy net $\left\{x_{\lambda}: \lambda \in \Lambda\right\}$ in $\left(N, \mathcal{D}_{N}\right)$ is also $I$-Cauchy in $(M, \mathcal{D})$ and if $(M, \mathcal{D})$ happens to be ideal complete space, so the net $\left\{x_{\lambda}: \lambda \in \Lambda\right\}$ is $I$-convergent to some $x_{0} \in M$ in $\left(M, \tau_{\mathcal{D}}\right)$. Now, if $x_{0} \in N$, then $\left\{x_{\lambda}: \lambda \in \Lambda\right\}$ is $I$-convergent in $\left(N, \tau_{\mathcal{D}_{N}}\right)$. But if $x_{0} \notin N$, then $\left\{x_{\lambda}: \lambda \in \Lambda\right\}$ is a net in $N \backslash\left\{x_{0}\right\}$ such that it is $I$-convergent to $x_{0} \in M$. Then, $x_{0}$ becomes a limit point of $N$ (by [13, Theorem 3]). Since $N$ is closed in $\left(M, \tau_{\mathcal{D}}\right)$, then $x_{0} \in N$. In any case, $x_{0} \in N$ if $N$ is closed in $\left(M, \tau_{\mathcal{D}}\right)$. Hence, $\left\{x_{\lambda}: \lambda \in \Lambda\right\}$ is an $I$-Cauchy net in $\left(N, \mathcal{D}_{N}\right)$ which becomes $I$-convergent in $\left(N, \tau_{\mathcal{D}_{N}}\right)$.

So, we conclude that $\left(C(X, Y), \mathcal{U}_{u}\right)$ is an ideal complete subspace of $\left(Y^{X}, \mathcal{U}_{u}\right)$.

If we involve the topology of $X$ in our function space and $Y$ has a uniform structure, we can have a uniform structure on $Y^{X}$ which is called the uniformity of uniform convergence on compacta or the uniformity of compact convergence. The definition of that uniformity and the associated topology is recalled below.

Definition 2.10 ([18). Suppose $Y$ has a uniformity $\mathcal{U}$. The uniformity of uniform convergence on compacta or the uniformity of compact convergence, $\mathcal{U}_{k}$, has for a subbase the sets

$$
E_{K, U}=\left\{(f, g) \in Y^{X} \times Y^{X}:(f(x), g(x)) \in U, \quad \text { for each } \quad x \in K\right\},
$$

where $K$ is a compact subset of $X$ and $U \in \mathcal{U}$. The topology $\tau_{\mathcal{U}_{k}}$ thus induced on $Y^{X}$ is the topology of compact convergence.

TheOREM 2.8. A net $\left\{f_{\lambda}: \lambda \in \Lambda\right\}$ is I-convergent to $f$ in $\left(Y^{X}, \tau_{\mathcal{U}_{k}}\right)$ where $\tau_{\mathcal{U}_{k}}$ is the topology of uniform convergence on compacta if and only if for each compact subset $K$ of $X,\left\{\left.f_{\lambda}\right|_{K}: \lambda \in \Lambda\right\}$ is uniformly I-convergent to $\left.f\right|_{K}$ in $\left(Y^{K}, \tau_{\mathcal{U}_{u}}\right)$, where $\tau_{\mathcal{U}_{u}}$ is the topology of uniform convergence on $Y^{K}$ and $I$ is a non-trivial ideal of $\Lambda$.

Proof. Let $\left\{f_{\lambda}: \lambda \in \Lambda\right\}$ be $I$-convergent to $f$ in $\left(Y^{X}, \tau_{\mathcal{U}_{k}}\right)$. For each subbasic open set $E_{K, U}[f]$ containing $f$ in $\left(Y^{X}, \tau_{\mathcal{U}_{k}}\right)$, this implies the set

$$
\left\{\lambda \in \Lambda: f_{\lambda} \notin E_{K, U}[f]\right\} \in I .
$$

This holds for each fixed compact subset $K$ of $X$ and for each $U \in \mathcal{U}$. Hence for each compact subset $K$ of $X,\left\{\left.f_{\lambda}\right|_{K}: \lambda \in \Lambda\right\}$ is uniformly $I$-convergent to $\left.f\right|_{K}$ in $\left(Y^{K}, \tau_{\mathcal{U}_{u}}\right)$. 


\section{I-COMPLETENESS IN FUNCTION SPACES}

Conversely, suppose $\left\{\left.f_{\lambda}\right|_{K}: \lambda \in \Lambda\right\}$ is uniformly $I$-convergent to $\left.f\right|_{K}$ in $\left(Y^{K}, \tau_{\mathcal{U}_{u}}\right)$ for each compact subset $K$ of $X$. Let $E_{K_{i}, U_{i}}\left[\left.f\right|_{K_{i}}\right]$ be arbitrarily chosen basic open sets containing $\left.f\right|_{K_{i}}$ in $\left(Y^{K_{i}}, \tau_{\mathcal{U}_{u}}\right)$ for $i=1,2, \ldots, n$, respectively. Then, we have the sets $\left\{\lambda \in \Lambda:\left.f_{\lambda}\right|_{K_{i}} \notin E_{K_{i}, U_{i}}\left[\left.f\right|_{K_{i}}\right]\right\} \in I$ for all $i=1,2, \ldots, n$. This implies the sets $\left\{\lambda \in \Lambda:\left.f_{\lambda}\right|_{K_{i}} \in E_{K_{i}, U_{i}}\left[\left.f\right|_{K_{i}}\right]\right\} \in F(I)$ for all $i=1,2, \ldots, n$, where $F(I)$ is the filter associated with the ideal $I$. We note below the follwing observation.

Let $K$ be any compact subset of $X, U$ be any member of the uniformity $\mathcal{U}$ on $Y$ and $f \in Y^{X}$. Now, we see that if $E_{K, U}\left[\left.f\right|_{K}\right]$ is a basic open set (as per Definition 2.7) containing $\left.f\right|_{K}$ in $\left(Y^{K}, \tau_{\mathcal{U}_{u}}\right)$ and $E_{K, U}[f]$ is a subbasic open set (as per Definition 2.10) containing $f$ in $\left(Y^{X}, \tau_{\mathcal{U}_{k}}\right)$, then

$$
\begin{aligned}
E_{K, U}\left[\left.f\right|_{K}\right] & =\left\{h \in Y^{K}:\left(\left.f\right|_{K}, h\right) \in E_{K, U}\right\} \\
& =\left\{h \in Y^{K}:\left(\left.f\right|_{K}(x), h(x)\right) \in U \text { for each } x \in K\right\}
\end{aligned}
$$

and

$$
\begin{aligned}
E_{K, U}[f] & =\left\{g \in Y^{X}:(f, g) \in E_{K, U}\right\} \\
& =\left\{g \in Y^{X}:(f(x), g(x)) \in U \text { for each } x \in X\right\} \\
& =\left\{g \in Y^{X}:\left(\left.f\right|_{K}(x),\left.g\right|_{K}(x)\right) \in U \text { for each } x \in K\right\} .
\end{aligned}
$$

Now, let $g \in Y^{X}$ be arbitrary. Then, $\left.g\right|_{K} \in Y^{K}$. Now, it is clear from the above that $\left.g\right|_{K} \in E_{K, U}\left[\left.f\right|_{K}\right]$ implies $g \in E_{K, U}[f]$, and conversely, $g \in E_{K, U}[f]$ implies $\left.g\right|_{K} \in E_{K, U}\left[\left.f\right|_{K}\right]$. Thus, we obtain

$$
\left\{\lambda \in \Lambda:\left.f_{\lambda}\right|_{K} \in E_{K, U}\left[\left.f\right|_{K}\right]\right\}=\left\{\lambda \in \Lambda: f_{\lambda} \in E_{K, U}[f]\right\} .
$$

Consequently, we get from the above observation that

$$
\left\{\lambda \in \Lambda: f_{\lambda} \in E_{K_{i}, U_{i}}[f]\right\} \in F(I) \quad \text { for all } i=1,2, \ldots, n .
$$

Hence,

$$
\begin{aligned}
& \bigcap_{i=1}^{n}\left\{\lambda \in \Lambda: f_{\lambda} \in E_{K_{i}, U_{i}}[f]\right\} \quad \in F(I), \\
\text { i.e., } \quad & \left\{\lambda \in \Lambda: f_{\lambda} \in \bigcap_{i=1}^{n}\left(E_{K_{i}, U_{i}}[f]\right)\right\} \in F(I), \\
\text { i.e., } & \left\{\lambda \in \Lambda: f_{\lambda} \in\left(\bigcap_{i=1}^{n} E_{K_{i}, U_{i}}\right)[f]\right\} \in F(I) .
\end{aligned}
$$

So, we get$$
\left\{\lambda \in \Lambda: f_{\lambda} \notin\left(\bigcap_{i=1}^{n} E_{K_{i}, U_{i}}\right)[f]\right\} \in I .
$$ 
Now, keeping in mind that $\left(\bigcap_{i=1}^{n} E_{K_{i}, U_{i}}\right)[f]$ being a basic open set in $\left(Y^{X}, \tau_{\mathcal{U}_{k}}\right)$ containing $f$ the net $\left\{f_{\lambda}: \lambda \in \Lambda\right\}$, is $I$-convergent to $f$ in $\left(Y^{X}, \tau_{\mathcal{U}_{k}}\right)$.

ThEOREM 2.9. A net $\left\{f_{\lambda}: \lambda \in \Lambda\right\}$ is an I-Cauchy net in $\left(Y^{X}, \mathcal{U}_{k}\right)$ where $\mathcal{U}_{k}$ is the uniformity of uniform convergence on compacta if and only if for each compact subset $K$ of $X,\left\{\left.f_{\lambda}\right|_{K}: \lambda \in \Lambda\right\}$ is uniformly I-Cauchy in $\left(Y^{K}, \mathcal{U}_{u}\right)$ where $\mathcal{U}_{u}$ is the uniformity of uniform convergence on $Y^{K}$ and $I$ is a non-trivial ideal of $\Lambda$.

Proof. Let $\left\{f_{\lambda}: \lambda \in \Lambda\right\}$ be an $I$-Cauchy net in $\left(Y^{X}, \mathcal{U}_{k}\right)$. Then, for each subbasic element $E_{K, U}$ there exists some $\lambda_{0} \in \Lambda$ such that the set

$$
\left\{\lambda \in \Lambda:\left(f_{\lambda}, f_{\lambda_{0}}\right) \notin E_{K, U}\right\} \in I .
$$

This holds for each fixed compact subset $K$ of $X$ and for each $U \in \mathcal{U}$. Hence, $\left\{\left.f_{\lambda}\right|_{K}: \lambda \in \Lambda\right\}$ is uniformly $I$-Cauchy in $\left(Y^{K}, \mathcal{U}_{u}\right)$ for each compact subset $K$ of $X$.

Conversely, let $\left\{\left.f_{\lambda}\right|_{K}: \lambda \in \Lambda\right\}$ be uniformly $I$-Cauchy in $\left(Y^{K}, \mathcal{U}_{u}\right)$ for each compact subset $K$ of $X$. Then, for arbitrarily chosen basic elements $E_{K_{1}, U_{1}}, E_{K_{2}, U_{2}}, \ldots$ $\ldots, E_{K_{n}, U_{n}}$ in $\left(Y^{K_{1}}, \mathcal{U}_{u}\right), \quad\left(Y^{K_{2}}, \mathcal{U}_{u}\right), \ldots,\left(Y^{K_{n}}, \mathcal{U}_{u}\right)$, respectively, we have by [4. Theorem 2.1] that there exist $A_{1}, A_{2}, \ldots, A_{n} \in I$ such that $\alpha, \beta \notin A_{i}$ implies $\left(\left.f_{\alpha}\right|_{K_{i}},\left.f_{\beta}\right|_{K_{i}}\right) \in E_{K_{i}, U_{i}}$ for each $i=1,2, \ldots, n$. We note below the following observation.

Let $K$ be any compact subset of $X, U$ be any member of the uniformity $\mathcal{U}$ on $Y$. Now, we see that if $E_{K, U}$ be a basic element for the uniformity $\mathcal{U}_{u}$ on $Y^{K}$ and a subbasic element for the uniformity $\mathcal{U}_{k}$ on $Y^{X}$, then for the first case mentioned

$$
\begin{aligned}
E_{K, U} & =\left\{(f, g) \in Y^{K} \times Y^{K}:(f(x), g(x)) \in U \text { for each } x \in K\right\} \\
& \subset\left\{(f, g) \in Y^{X} \times Y^{X}:(f(x), g(x)) \in U \text { for each } x \in K\right\}=E_{K, U}
\end{aligned}
$$

mentioned for the second case.

Thus, we obtain that $\left(\left.f_{\alpha}\right|_{K},\left.f_{\beta}\right|_{K}\right) \in E_{K, U}$ implies $\left(f_{\alpha}, f_{\beta}\right) \in E_{K, U}$. Let us say $A=\bigcup_{i=1}^{n} A_{i}$. Then, clearly $A \in I$, and it follows from the observation made just before that $\alpha, \beta \notin A$ implies $\left(f_{\alpha}, f_{\beta}\right) \in E_{K_{i}, U_{i}}$ for all $i=1,2, \ldots, n$. Hence, we can conclude that $\alpha, \beta \notin A$ implies $\left(f_{\alpha}, f_{\beta}\right) \in \bigcap_{i=1}^{n} E_{K_{i}, U_{i}}$. Since $\bigcap_{i=1}^{n} E_{K_{i}, U_{i}}$ is a basic element for the uniformity $\mathcal{U}_{k}$ on $Y^{X}$, so, it follows by [4, Theorem 2.1] that $\left\{f_{\lambda}: \lambda \in \Lambda\right\}$ is an $I$-Cauchy net in $\left(Y^{X}, \mathcal{U}_{k}\right)$.

We now recall the concept of a topological space to be a $k$-space which plays a central role in the discussion of both completeness and compactness relative to the uniformity of uniform convergence on compacta and its topology. 


\section{I-COMPLETENESS IN FUNCTION SPACES}

Definition 2.11 ([18]). A topological space $(X, \tau)$ is a $k$-space (or a compactly generated space) if and only if the following condition holds:

(a) $A \subset X$ is open in $(X, \tau)$ if and only if $A \cap K$ is open in $\left(K, \tau_{K}\right)$ for each compact set $K$ in $(X, \tau)$.

The $k$-spaces are important to our discussion of $I$-convergence of continuous functions on compacta because, in these spaces, the continuous functions are precisely those which behave well on compact subsets. The proof of the following lemma, which says this more precisely, follows easily in applying the definition of a $k$-space.

LEMma 2.10 ([18]). If $X$ is a $k$-space and $Y$ is a topological space, then $f: X \rightarrow Y$ is continuous if and only if $\left.f\right|_{K}$ is continuous for each compact $K \subset X$.

Using this result and Theorems 2.8 and 2.9, which describes $I$-convergence on compacta as being precisely uniform $I$-convergence on each compact subset, the following theorem holds good.

TheOREM 2.11. If $X$ is a $k$-space and $(Y, \mathcal{U})$ is an ideal complete uniform space, then $C(X, Y)$ is ideal complete in the uniformity of uniform convergence on compacta.

P r o o f. At first, from Theorem 2.9 we know that if $\left\{f_{\lambda}: \lambda \in \Lambda\right\}$ is an $I$-Cauchy net in $\left(Y^{X}, \mathcal{U}_{k}\right)$ where $\mathcal{U}_{k}$ is the uniformity of uniform convergence on compacta, then we have $\left\{\left.f_{\lambda}\right|_{K}: \lambda \in \Lambda\right\}$ is uniformly $I$-Cauchy in $\left(Y^{K}, \mathcal{U}_{u}\right)$ for each compact subset $K \subset X$, where $\mathcal{U}_{u}$ is the uniformity of uniform convergence on $Y^{K}$ and $I$ is a non-trivial ideal of $\Lambda$. On the other hand, from Theorem 2.7 we know that for each compact $K \subset X, C(K, Y)$ is an ideal complete subspace of $Y^{K}$ in the uniformity of uniform convergence. Now, let $\left\{f_{\lambda}: \lambda \in \Lambda\right\}$ be an $I$-Cauchy net in $\left(C(X, Y), \mathcal{U}_{k}\right)$. Then, $\left\{\left.f_{\lambda}\right|_{K}: \lambda \in \Lambda\right\}$ is uniformly $I$-Cauchy in $\left(C(K, Y), \mathcal{U}_{u}\right)$ for each compact $K \subset X$. Since $\left(C(K, Y), \mathcal{U}_{u}\right)$ is ideal complete, so, a continuous uniform $I$-limit $f_{K}: K \rightarrow Y$ exists for each compact $K \subset X$. It can be seen easily that if $K_{1} \subset K_{2} \subset X$, then $\left.f_{K_{2}}\right|_{K_{1}}=f_{K_{1}}$, and from this it follows that the function $f: X \rightarrow Y$ defined by $f(x)=f_{K}(x)$ for $x \in K$, is well defined. It is continuous by above Lemma 2.10, and since $\left\{\left.f_{\lambda}\right|_{K}: \lambda \in \Lambda\right\}$ is uniformly $I$-convergent to $\left.f\right|_{K}$ in $\left(Y^{K}, \tau_{\mathcal{U}_{u}}\right)$ for each compact $K \subset X$, so, by Theorem 2.8 it follows that $\left\{f_{\lambda}: \lambda \in \Lambda\right\}$ is $I$-convergent to $f$ in $\left(Y^{X}, \mathcal{U}_{k}\right)$. Hence, we get that $\left\{f_{\lambda}: \lambda \in \Lambda\right\}$ is $I$-convergent to $f$ in $\left(C(X, Y), \mathcal{U}_{k}\right)$. Thus, $\left(C(X, Y), \mathcal{U}_{k}\right)$ is ideal complete.

Acknowledgement. The second author is thankful to The University Grants Commission, Government of India, for giving the award of Senior Research Fellowship during the tenure of preparation of this research paper. 


\section{AMAR KUMAR BANERJEE - APURBA BANERJEE}

\section{REFERENCES}

[1] BALÁŽ, V.-ČERVEŇANSKÝ, J.-KOSTYRKO, P.—ŠALÁT, T.: I-convergence and I-continuity of real functions, Acta Mathematica 5 (2002), 43-50.

[2] BANERJEE, A. K.-BANERJEE, A.: A note on I-convergence and $I^{*}$-convergence of sequences and nets in topological spaces, Mat. Vesnik 67(2015), 212-221.

[3] DEMIRCI, K.: I-limit superior and limit inferior, Math. Commun. 6 (2001), 165-172.

[4] DAS, P.-GHOSAL, S.K.: On I-Cauchy nets and completeness, Topology Appl. 157 (2010), 1152-1156.

[5] FAST, H.: Sur la convergence statistique, Colloq. Math. 2 (1951), 241-244.

[6] HAlBeRSTEM, H.-ROTH, K. F.: Sequences. Springer, New York, 1993.

[7] KELLEY, J. L.: General Topology. (2nd ed.). In: Grad. Texts in Math. Vol. 27, Springer-Verlag, Berlin, 1975.

[8] KOSTYRKO, P.-ŠALÁT, T.-WILCZYŃSKI, W.: I-convergence, Real Anal. Exchange $26(2000 / 2001), 669-686$.

[9] KOSTYRKO, P.-MAČAJ, M.-ŠALÁT, T.-SLEZIAK, M.: I-convergence and extremal I-limit point, Math. Slovaca 55 (2005), 443-464.

[10] KURATOWSKI, K.: Topologie I. PWN, Warszawa, 1961.

[11] LAHIRI, B. K.-DAS, P.: Further results on I-limit superior and I-limit inferior, Math. Commun. 8 (2003), 151-156.

[12] _I I and $I^{*}$-convergence in topological spaces, Math. Bohemica 130 (2005), 153-160.

[13] _I and $I^{*}$-convergence of nets, Real Anal. Exchange 33 (2007/2008), 431-442.

[14] MAČAJ, M.- ŠALÁT, T.: Statistical convergence of subsequences of a given sequence, Math. Bohemica 126 (2001), 191-208.

[15] NIVEN, I-ZUCKERMAN, H. S.: An Introduction to the Theory of Numbers. (4th ed.). John Wiley, New York, 1980.

[16] ك̆ALÁT, T.: On statistically convergent sequences of real numbers, Math. Slovaca 30 (1980), 139-150.

[17] SCHOENBERG, I. J.: The integrability of certain functions and related summability methods, Amer. Math. Monthly 66 (1959), 361-375.

[18] WILlARD, S.: General Topology. Dover Publ., Inc., Mineola, NY, USA, 2004.

Received May 15, 2017

\author{
Department of Mathematics \\ The University of Burdwan \\ Burdwan-713104 \\ West Bengal \\ INDIA \\ E-mail: akbanerjee@math.buruniv.ac.in \\ akbanerjee1971@gmail.com \\ apurbabanerjeemath@gmail.com
}

